\title{
GNNQQNY: Methodology for biophysical and structural understanding of aggregation
}

Gunasekhar Burra ${ }^{1,2,4 *}$, Mahmoud B. Maina ${ }^{2,3}$, Louise C. Serpell ${ }^{2 *}$ and Ashwani K. Thakur $^{1 *}$

${ }^{1}$ Department of Biological Sciences and Bioengineering, Indian Institute of Technology Kanpur, India.

${ }^{2}$ Sussex Neuroscience, School of Life Sciences, University of Sussex, UK.

${ }^{3}$ College of Medical Sciences, Yobe State University, Nigeria.

${ }^{4}$ Current address: Analytical Research \& Development, Pharma Division, Biological E. Limited, Genome Valley, IKP-Shameerpet, Hyderabad - 500 078, Telangana, India.

\section{*Correspondence:}

Gunasekhar Burra: Analytical Research \& Development, Pharma Division, Biological E. Limited, Genome Valley, IKP-Shameerpet, Hyderabad - 500 078, Telangana, India. Email:gunasekhar.iitk@gmail.com.Contact:+91-8869970001.

Louise C. Serpell: Sussex Neuroscience, School of Life Sciences, University of Sussex, UK. Email: L.C.Serpell@sussex.ac.uk. Contact: +44-1273 877363.

Ashwani K. Thakur: Department of Biological Sciences and Bioengineering, Indian Institute of Technology Kanpur, India. E-mail: akthakur@iitk.ac.in. Contact: +91-512-259-4077.

Keywords: Solubilization; pH; Nucleation kinetics; Aggregation; Amyloid; Seeding

Abbreviations: CR, Congo red; ESI, Electrospray ionization; FPLC, Fast protein liquid chromatography; FTIR, Fourier-transform infrared spectroscopy; $\mathrm{HCl}$, Hydrogen chloride; LS, Light scattering; PBS, Phosphate-buffered saline; RP-HPLC, Reversed-phase high performance liquid chromatography; SEC, Size exclusion chromatography; TEM, Transmission electron microscopy; ThT, Thioflavin T; ToF, Time-of-flight.

Running title: Reproducible solubilisation method for GNNQQNY 


\begin{abstract}
GNNQQNY sequence offers crucial information about the formation and structure of an amyloid fibril. In this study, we demonstrate a reproducible solubilisation protocol where the reduction of $\mathrm{pH}$ to 2.0 resulted in the generation of GNNQQNY monomers. The subsequent ultracentrifugation step removes the residual insoluble peptide from the homogeneous solution. This procedure ensures and allows the peptides to remain monomers till their aggregation is triggered by adjusting the $\mathrm{pH}$ to 7.2. The aggregation kinetics analysis showed a distinct lag-phase that is concentration-dependent, indicating nucleationdependent aggregation kinetics. Nucleation kinetics analysis suggested a critical nucleus of size $\sim 7$ monomers at physiological conditions. The formed nucleus acts as a template for further self-assembly leading to the formation of highly ordered amyloid fibrils. These findings suggest that the proposed solubilisation protocol provides the basis for understanding the kinetics and thermodynamics of amyloid nucleation and elongation in GNNQQNY sequences. This procedure can also be used for solubilising such small amyloidogenic sequences for their biophysical studies.
\end{abstract}




\section{Introduction}

Peptide fragments are widely used for physico-chemical understanding of the otherwise complex mechanism of in vivo protein aggregation[1,2]. The relatively lower complexities offered by these fragments makes them ideal models for in vitro aggregation studies[3-5]. The N-terminal ${ }^{7}$ GNNQQNY ${ }^{13}$ sequence of Sup35 protein serves to be useful for understanding the biophysical and structural aspects of amyloids[6]. X-ray fibre diffraction analysis of amyloid-like nano-crystals and fibrils formed by GNNQQNY showed characteristic reflections of a cross- $\beta$ spine containing 'Steric-zipper' structure[3, 7-12]. Significant progress was achieved so far in understanding the molecular architecture of mature amyloid fibers formed by GNNQQNY, which were further complemented by simulation studies[13, 14]. But, the early steps of in vitro kinetic and thermodynamic changes during its self-assembly are yet to be elucidated.

Much of the information regarding the early steps of amyloid formation by GNNQQNY comes from the computer simulation studies. These studies elucidated the thermodynamic and kinetic details associated with the transition of GNNQQNY monomers to oligomers to mature fibrils[13-21]. Some studies even suggested that there exists an enthalpic barrier step, pointing towards the nucleation-dependent aggregation mechanism through which it forms fibrils rich in $\beta$-sheets $[2,10,11,13,15-18,22,23]$. This involves the formation of a metastable critical nucleus within the monomer pool along the path of aggregation. The formed nucleus acts as a template for further monomer addition at a much faster rate leading to the elongation of amyloid fibrils[24-27].

Nelson et al (2005) predicted that 3-4 GNNQQNY monomers constitute the metastable nucleus based on theoretical energetics[10]. Molecular dynamic simulations study by Nasica-Labouze and Mousseau (2012) suggested a nucleus of size about 4-5 monomers and 
5-6 monomers at $280 \mathrm{~K}$ and $300 \mathrm{~K}$ temperature, respectively[23]. In a recent study, SzałaMendyk and Molski (2020) suggested a relatively larger critical nucleus of size $\sim 25$ monomers[20]. In contrast to these observations, Langkilde et al (2015) observed lack of intermediates during the aggregation process and thus suggested that the elongation process is likely to occur via monomer addition[28]. However, these studies will have practical implications only when they are proven through experimental evidences.

Although significant progress was achieved in understanding the molecular architecture of mature GNNQQNY amyloid fibers, the early steps of in vitro kinetics and thermodynamics of its assembly still need to be elucidated. This is probably due to the lack of a reproducible solubilisation procedure that ensures the homogenous monomeric solution to begin with. Usually the presence of insoluble aggregates due to improper solubilisation acts as templates for further monomer addition and the variation in the starting conformations leads to conflicting results[29, 30].

To overcome this issue, solvent-based pre-processing methods were usually employed to improve the solubility of proteins and amyloidogenic sequences such as amyloid- $\beta$ (A $\beta)$, polyglutamine-containing polypeptides, insulin amyloid polypeptide (IAPP), and etc[29-39]. These methods use different solvents such as trifluoroacetic acid (TFA), Dimethyl sulfoxide (DMSO), trifluoroethanol (TFE), hexafluoroisopropanol (HFIP) and ammonium hydroxide. Our recent study on polyglutamine-containing polypeptides showed that the organic solvents, TFA and HFIP aid in solubilisation by promoting the exchange of strong and intricate side chain-side chain and side chain-main chain hydrogen bonds present within the lyophilized peptides with themselves[30]. Similar observations were reported later by Jakubek et al (2019) as well based on UV resonance Raman spectroscopy analysis[40]. 
In this study, we demonstrate a solubilisation protocol that generates homogenous monomeric solution reproducibly. This enabled the study of aggregation and nucleation kinetics analyses of GNNQQNY by quantitative RP-HPLC-based sedimentation assay. The data convincingly suggested nucleation-dependent aggregation kinetics for GNNQQNY. The nucleation kinetics analysis under physiological conditions $\left(\mathrm{pH} 7.4\right.$ and $\left.37{ }^{\circ} \mathrm{C}\right)$ resulted in a critical nucleus of size seven monomers. The mature fibrils were long unbranched structures and stained positively for ThT and Congo-red dyes. They were also found to be non-toxic and thus caused no significant cell death as compared to controls.

\section{Materials and Methods}

\section{$\underline{\text { Materials }}$}

Pure form of GNNQQNY (>95\%), Trifluoroacetic acid (TFA), formic acid, Congo-red and thioflavin $\mathrm{T}$ were obtained from Sigma Aldrich. HPLC-grade acetonitrile (ACN) and hydrochloric acid $(\mathrm{HCl})$ were purchased from Merck and sodium-azide was procured from SD fine chemicals Ltd. Phosphate-buffered saline (PBS) was prepared as per Cold Spring Harbor protocol by dissolving $\mathrm{Na}_{2} \mathrm{HPO}_{4}(7.2 \mathrm{~g}), \mathrm{KH}_{2} \mathrm{PO}_{4}(1.2 \mathrm{~g}), \mathrm{NaCl}(40 \mathrm{~g})$ and $\mathrm{KCl}(1 \mathrm{~g})$ procured from Merck.

\section{$\underline{\text { Solubilisation of peptides }}$}

GNNQQNY peptide was solubilised at $2 \mathrm{mg} / \mathrm{mL}$ concentration in milliQ-water acidified to pH 2.0 using TFA or $\mathrm{HCl}$. The solution was gently swirled till the white precipitate disappears to ensure complete solubilisation of the peptide. The resultant solution was then subjected to ultracentrifugation for a minimum of $30 \mathrm{~min}$ to $2 \mathrm{~h}$ at $80,000 \mathrm{RPM}$ and $25{ }^{\circ} \mathrm{C}$. Carefully the top $2 / 3^{\text {rd }}$ of the supernatant was taken, leaving behind the bottom $1 / 3^{\text {rd }}$ of the solution which may contain the insoluble aggregates. This supernatant was used for carrying the biophysical and aggregation analysis reported in this study. 


\section{Mass spectrometry}

The $m / z$ spectrum of soluble GNNQQNY peptides was recorded by injecting $5 \mu \mathrm{L}$ of sample into time-of-flight (TOF) mass analyser equipped with electrospray ionization (ESI) source (Waters Q-TOF premier HAB213). The data was plotted at a mass range of 50-3500 Da using OriginPro 8.5 data analysis and graphing software. The purity and solubility was determined by analysing all the observed molecular ionization species of the peptide[41].

\section{Concentration determination by Reversed-Phase HPLC}

GNNQQNY peptide stock solution $(0.4 \mathrm{mg} / \mathrm{mL})$ was prepared by solubilising as previously mentioned and used for determining the standard curve. The stock was diluted serially and the optical density (OD) of each dilution was determined at $220 \mathrm{~nm}$ by using NanoDrop ND-1000 UV-Vis Spectrophotometer. The corresponding concentration $(\mu \mathrm{g})$ was back calculated based on the standard procedure suggested by Kuipers and Gruppen (2007)[29, $30,42,43]$. Briefly, the molar extinction coefficient used for determining the concentration was obtained by summing up the molar extinction coefficients of all the amino acids and the peptide bonds at $214 \mathrm{~nm}$ [42]. Each of these dilutions was subjected to RP-HPLC analysis by passing through Agilent eclipse plus $\mathrm{C}_{18}$ column $(4.6 \mathrm{~mm} \times 100 \mathrm{~mm})$ connected to Agilent 1260 Infinity Quaternary system. A gradient flow of water and acetonitrile containing $0.05 \%(\mathrm{v} / \mathrm{v})$ TFA at a rate of $1 \mathrm{~mL} / \mathrm{min}$ was used for elution. The standard-curve was obtained by plotting the area under the curve determined at $214 \mathrm{~nm}$ for each of the dilution against the respective concentration $(\mu \mathrm{g})$ calculated based on OD. The data was subjected to linear curve-fit using OriginPro 8.5 graphing tool and the unknown concentration $(\mu \mathrm{g})$ of the peptide was determined by fitting the area in the standard curve.

\section{$\underline{\text { Analytical size-exclusion chromatography (SEC) }}$}


The $\mathrm{pH}$ of $306 \mu \mathrm{M}$ of soluble GNNQQNY peptide in water-HCl (pH 2.0) was adjusted to pH 7.4 by using PBS. $100 \mu \mathrm{L}$ of this solution was analysed using SEC method. For this, the sample was passed through Superdex peptide 10/300GL column (GE healthcare); connected to Biologic Duoflow model Bio-Rad fast protein liquid chromatography (FPLC) system. PBS ( $\mathrm{pH} 7.4$ ) was run at a flow rate of $0.5 \mathrm{~mL} / \mathrm{min}$ to monitor the elution profile of the peptide. The absorbance was recorded at $215 \mathrm{~nm}$ while maintaining a constant pressure of $\sim 80 \operatorname{psi}[30,41]$.

\section{$\underline{\text { Aggregation kinetics analysis }}$}

The aggregation kinetics reactions were monitored in PBS at $\mathrm{pH} 7.4$ and $37^{\circ} \mathrm{C}$. Sodiumazide $(0.05 \%)$ was added to the reaction mixture to prevent microbial contamination[29, 34 , $44,45]$. The rate of aggregation was monitored by measuring the monomer concentration as a function of time by using sedimentation assay. For this, an aliquot of sample was taken from the ongoing aggregation reaction at regular time intervals and subjected to ultracentrifugation at $25,000 \mathrm{rcf}$ and $25{ }^{\circ} \mathrm{C}$ for $30 \mathrm{~min}$. The supernatant was taken and adjusted to $20 \%$ formic acid before injecting into RP-HPLC. The monomer concentration was determined based on the above standard curve. Addition of formic acid was shown to slowdown aggregation as it reduces the $\mathrm{pH}$ of the sample significantly[34].

The sedimentation assay data was complimented by monitoring the rate of formation of higher order structures by using light scattering (LS) and Thioflavin T (ThT) assays[46, 47]. These assays were performed by taking $120 \mu \mathrm{L}$ of ongoing aggregation reaction sample at different time points in a Quartz SUPRASIL Ultra-micro cell[30]. The emission and excitation wavelengths were set at $450 \mathrm{~nm}$, and slit widths at $2.5 \mathrm{~nm}$ for determining the intensity of scattered light. To this, $2.5 \mathrm{mM}$ ThT stock was added to a final concentration of $100 \mu \mathrm{M}$ and the excitation and emission wavelengths were reset to $450 \mathrm{~nm}$ (slit width, 5 
$\mathrm{nm}$ ) and $489 \mathrm{~nm}$ (slit width, $5 \mathrm{~nm}$ ), respectively to measure the ThT intensity. These assays were performed using LS 55 spectrofluorimeter (Perkin Elmer). Three consecutive spectra were averaged after blank correction for obtaining the final LS and ThT spectra.

\section{$\underline{\text { Seeding assay }}$}

Seeding reactions were monitored in PBS ( $\mathrm{pH} 7.4)$ at $37^{\circ} \mathrm{C}$ by adding the preformed seeds $(2 \%, \mathrm{wt} / \mathrm{wt})$ grown at similar conditions as described previously[29, 48]. The control reactions used for comparison were monitored without addition of preformed seeds. The drop in the monomer concentration with time was determined by using sedimentation assay. The aggregation kinetics of both the conditions was compared by plotting the monomer percentages against time (h) by using OriginPro 8.5 software.

\section{Nucleation Kinetics analyses}

Different concentrations of freshly solubilised GNNQQNY were incubated in PBS at pH 7.4 and $37^{\circ} \mathrm{C}$. The drop in monomer concentration with time was measured at different time intervals using sedimentation assay. Monomer concentration $(\mu \mathrm{M})$ versus time ${ }^{2}\left(\mathrm{~s}^{2}\right)$ graphs were plotted for each concentration by collecting as many data points as possible within $20 \%$ drop in monomer concentration. Using the slopes of these time ${ }^{2}$ graphs, $\log [$ initial concentration $(\mathrm{M})$ ] versus $\log [$ slope] plots were constructed. This plot was subjected to linear curve-fit and the obtained slope was fit in the equation, slope $=n^{*}+2$, where $n^{*}$ indicates the size of the critical nucleus[24, 29, 34, 48, 49].

\section{$\underline{\text { Thioflavin T (ThT) and Congo-red staining of fibrils }}$}

Standard solutions of ThT $(0.8 \mathrm{mg} / \mathrm{mL})$ and Congo-red $(1 \mathrm{mg} / \mathrm{mL})$ prepared in MilliQ-water and filtered through $0.2 \mu \mathrm{m}$ filter were used to stain the GNNQQNY fibers. $10 \mu \mathrm{L}$ of peptide solution was evenly spread on a neat glass slide and allowed to air-dry. A drop of filtered ThT and Congo-red solution was added on the dry peptide film and incubated for 30 
min. Excess dye was removed by washing with MilliQ-water followed by air drying for 30 min. Bright field images and the associated ThT fluorescence and Congo-red birefringence images were recorded using appropriate light filters on Leica DM2500 model fluorescent microscope[3, 50].

Fourier-transform infrared (FTIR) spectroscopy

Soluble form of GNNQQNY and the fibril suspension were analysed using Bio-ATR II cell mounted on Bruker Tensor-27 FTIR instrument[29, 30, 51]. $300 \mu \mathrm{L}$ of fully grown GNNQQNY fibers were washed thrice by using milliQ-water and then concentrated to 100 $\mu \mathrm{L} .50 \mu \mathrm{L}$ of sample was loaded on to the Bio-ATR II cell for analysis. The solid-state FTIR analysis was performed by loading the KBr-mixed GNNQQNY powder on to MIRATR cell. The final FTIR spectrum was an average of 120 scans at a resolution of $4 \mathrm{~cm}^{-1}$. The spectrum was auto-corrected for water vapour and the blank. The second derivative of primary spectra was reported after nine-point smoothing by Opus 7.2 spectroscopy software (Bruker Optics).

\section{Transmission electron microscopy (TEM)}

Samples were prepared by inverting the carbon-coated side of size 300 mesh copper TEM grid on $5 \mu \mathrm{L}$ of aggregate sample for thirty seconds. The excess sample was carefully blotted by using KimWipes and was rinsed with $10 \mu \mathrm{L}$ of milliQ-water followed by inverting on $5 \mu \mathrm{L}$ of $2 \%$ uranyl acetate for $5-10$ seconds[29, 30, 52]. The stained grids were allowed overnight to air-dry and the electron micrographs of fibrils were recorded using FEI-Tecnai G1 12 Twin (120 KV) transmission electron microscope.

\section{$\underline{\text { X-ray fiber diffraction }}$}


$200 \mu \mathrm{L}$ of GNNQQNY peptide $(10 \mathrm{mg} / \mathrm{mL})$ was incubated at $37{ }^{\circ} \mathrm{C}$ for several days to generate fibers. Approximately $20 \mu \mathrm{L}$ of this sample was placed and allowed to air-dry overnight between two wax-filled capillaries. The generated fiber was placed on a goniometer head mounted on a Rigaku X-ray diffractometer (Sevenoaks, UK) equipped with rotating anode $(\mathrm{CuK} \alpha)$ and Raxis IV++ detector. The specimen-to-film distance of 50 $\mathrm{mm}$ or $100 \mathrm{~mm}$ was set for recording the data[53].

\section{$\underline{\text { Cytotoxicity and Cell viability assays }}$}

Undifferentiated SHSY5Y cells were differentiated using established protocol[54, 55]. The differentiated cells were incubated with GNNQQNY fibers ( $5 \mu \mathrm{M}$ and $20 \mu \mathrm{M})$ for 48 hours. Phosphate-buffered saline $(1 \mathrm{X})$ and $\mathrm{H}_{2} \mathrm{O}_{2}(1 \mathrm{mM})$ were used as negative and positive controls, respectively. Towards the end of the incubation, the cells were treated with $5 \mu \mathrm{M}$ CellROX Green Reagent (C10444, Life technologies UK) for 45 min to evaluate the level of oxidative stress. This was followed by incubation with ReadyProbes reagent (Life Technologies) for 15 min to evaluate cell death. The cells were imaged at $37^{\circ} \mathrm{C}$ and $5 \% \mathrm{CO}_{2}$ on Operetta CLS high-content imaging system (PerkinElmer) by using DAPI, FITC and TRITC filters. To ensure the reproducibility of the findings, three independent experiments each containing a minimum of 5000 cells were analysed using Harmony software automated analysis algorithm provided within the system[56].

\section{Results and discussion}

The amyloidogenic sequences such as GNNQQNY poses a challenge to unambiguously resolve the kinetic and thermodynamic properties associated with its aggregation. This is mainly due to improper or partial solubilisation leading to contamination of the aggregation reaction with pre-aggregated peptide. Existence of such forms in the starting reaction results in spontaneous aggregation which is often non-reproducible owing to the heterogeneous nature of the starting material. Probability of this phenomenon was explored by analysing 
the infrared spectra of GNNQQNY powder and its solubilised form (figure 1A). Solid-state infrared spectra of lyophilized GNNQQNY powder showed peaks matching to parallel $\left(1634 \mathrm{~cm}^{-1}\right.$ and $\left.1682 \mathrm{~cm}^{-1}\right)$ and anti-parallel $\left(1623 \mathrm{~cm}^{-1}\right.$ and $\left.1689 \mathrm{~cm}^{-1}\right) \beta$-sheets and random coil $\left(1648 \mathrm{~cm}^{-1}\right)$ conformations (figure $\left.1 \mathrm{~A}\right)[30,57,58]$. The side chain $\mathrm{NH}_{2}$ bending $(1610$ $\left.\mathrm{cm}^{-1}\right)$ and $\mathrm{C}=\mathrm{O}$ stretching $\left(1656 \mathrm{~cm}^{-1}\right.$ and $\left.1669 \mathrm{~cm}^{-1}\right)$ vibrations of $\mathrm{Gln} /$ Asn residues were also identified (figure 1A)[59]. The solid form of GNNQQNY also showed two more prominent peaks at $1698 \mathrm{~cm}^{-1}$ and $1706 \mathrm{~cm}^{-1}$, indicating the presence of weaker H-bond interactions in the lyophilised forms (figure 1A)[30, 59]. Overall, this data suggests that the lyophilised form of GNNQQNY is an amorphous aggregate that may or may not be soluble in aqueous solution[30].

Unlike other amyloidogenic sequences such as $A \beta$, polyglutamines and IAPP that require special solvent pre-treatment to enhance solubility, GNNQQNY was freely soluble in water and the resultant solution was clear and transparent. When this solution was analysed using Bio-ATR IR, the characteristic bands indicating the presence of $\beta$-sheets $\left(1623 \mathrm{~cm}^{-1}, 1634\right.$ $\mathrm{cm}^{-1}, 1682 \mathrm{~cm}^{-1}$ and $1689 \mathrm{~cm}^{-1}$ )in the lyophilised forms were greatly diminished (figure 1A) $[30,57,59]$. In addition, the emergence of new bands suggesting a more unordered conformation $\left(1628 \mathrm{~cm}^{-1}, 1640 \mathrm{~cm}^{-1}\right.$ and $1677 \mathrm{~cm}^{-1}$ ) were observed (figure 1A)[30, 57, 59]. The structural transition of $\beta$-sheets in solid form to unordered conformation in solution further confirms solubility of GNNQQNY sequences. The probable reasons for such easy transition of conformations might be the dominance of relatively less stable parallel $\beta$-sheets due to longer and less optimal H-bonds and the existence of weaker H-bond interactions that are easily accessible to the solvent $[60,61]$.

However, the major issue is that the GNNQQNY sequences solubilised in pure water tends to spontaneously aggregate, and thus result in non-homogeneous solution by the time the aggregation reactions are initiated. This is probably due to the fact that the isoelectric point 
of GNNQQNY (pI, 5.9) is close to that of the water (pI, 6.5-7.0), and that might promote its spontaneous aggregation or precipitation. One way to overcome this phenomenon is by maintaining the $\mathrm{pH}$ of the solution at a point that is far away from the $\mathrm{pI}$ of GNNQQNY. This can be achieved by either significantly increasing or decreasing the $\mathrm{pH}$ from 5.9 . Usually, the peptides/proteins are positively charged at a $\mathrm{pH}$ below their $\mathrm{pI}$ and are negatively charged at a $\mathrm{pH}$ above their $\mathrm{pI}$ depending on their sequence[60,61]. This property was taken advantage here and the $\mathrm{pH}$ of the solvent (milliQ water) was significantly reduced to 2.0 by using $\mathrm{HCl}$ or TFA. This point was considered owing to the fact that five out of the seven residues in GNNQQNY are polar in nature. At such low pH (2.0), the side-chains of asparagine $(\mathrm{N})$ and glutamine $(\mathrm{Q})$ residues are highly positively charged and thus repel with each other, thereby aiding in their solubility through interaction with water[43, 62].

Further, the homogeneous nature of the monomeric solution was ensured by subjecting the solubilised solution to ultracentrifugation (80,000 RPM) for at least $30 \mathrm{~min}$ at $25{ }^{\circ} \mathrm{C}$. We have shown recently in one of our studies that temperature plays a prominent role in regulating the aggregation of GNNQQNY[43]. Particularly, the efficiency of nucleation was found to be very high at low temperatures[43]. Hence the ultracentrifugation at low temperatures and the storage of solubilised peptide under freezing conditions should be avoided for better reproducibility of results. The purity, identity and monomeric state of GNNQQNY were confirmed by native ESI-MS analysis where only the expected $\mathrm{m} / \mathrm{z}$ value of monomer (836.4 Da) was observed (figure 1B). Subjecting the supernatant to analytical SEC analysis also resulted in a single peak, thus confirming the homogenous nature of the solution (figure 1C). Absence of additional peaks and the elution of GNNQQNY (836.4 Da) before sodium azide $\left(\mathrm{NaN}_{3}, 65 \mathrm{Da}\right)$ and after MATLEKLMKAFESLKSF (1974.4 Da) further confirmed the monomeric state of the solubilised fraction (figure 1C). 
The lack of an aromatic amino acid residue tryptophan in GNNQQNY sequence makes it difficult to monitor the aggregation kinetics based on quantitative methods like analytical reversed-phase high performance liquid chromatography (RP-HPLC). The data generated by Thioflavin $\mathrm{T}$ (ThT) and light scattering assays has no practical meaning as it is qualitative in nature. Hence we took the work of Kuirper and Gruppen as basis and developed a quantitative method to follow the aggregation kinetics. In this method, the overall contribution of the molar extinction coefficients of individual amino acids and the peptide bond at $215 \mathrm{~nm}$ was taken into consideration for determining the concentration[42]. Upon plotting the concentration against the respective area under the curve obtained from RPHPLC for different dilutions at $215 \mathrm{~nm}$, we obtained a standard curve (figure 2A)[42]. This standard curve was used for determining the drop in monomer concentration during aggregation kinetics analysis.

We then tested the ability of this solubilisation method to understand the aggregation kinetics and reproduce the data reported in the literature by adjusting the $\mathrm{pH}$ of the sample to 7.4 at $37^{\circ} \mathrm{C}$. The rate of aggregation was monitored by measuring the drop in monomer concentration as a function of time by using sedimentation assay. This data was further supplemented by measuring the rate of formation of aggregates with time by using ThT and light scattering assays. The sedimentation assay data clearly showed a lag-phase of $\sim 12 \mathrm{~h}$, where the drop in monomer concentration was negligible and took about $25 \mathrm{~h}$ for an initial drop in 20\% monomer (figure 2B). Collectively this data indicated a lag-phase of 20-25 h for GNNQQNY $(1253 \pm 12 \mu \mathrm{M})$, within which the energetically unfavourable nuclei are formed. This was followed by a rapid decrease in the concentration of monomer, indicating an elongation-phase. No further drop in monomer concentration was observed towards the end of elongation-phase, and is suggestive of an equilibrium or stationary-phase (figure 2B). The concentration of monomer at the equilibrium-phase is usually termed as the critical 
concentration $\left(\mathrm{C}_{\mathrm{r}}\right)$, below which the efficiency of nucleation is negligible. Overall, this data hinted at a nucleation-dependent aggregation mechanism for GNNQQNY[34, 49, 63, 64].

However, a classical nucleation-dependent aggregation kinetics should also display; seeding effect, specific ThT fluorescence indicating critical nucleus formation, and concentrationdependence of rate of aggregation[34,65]. The effect of seeding on aggregation was tested by adding the preformed seeds $(2 \%, \mathrm{wt} / \mathrm{wt})$ at the start of the reaction. Addition of seeds eliminated the lag-phase by spontaneously reducing the monomer concentration to $25 \%$ within 3-4 $\mathrm{h}$ of incubation (figure $2 \mathrm{~B}$ ). This is mainly because the added aggregates act as templates for monomer addition, thereby drives the aggregation via the elongation-phase[29, 34, 63-67].

In agreement with the sedimentation assay data, an enhancement in ThT fluorescence was observed only after $12 \mathrm{~h}$ of incubation. The binding of ThT to aggregates usually results in enhanced fluorescence at $489 \mathrm{~nm}[68-70]$. This data confirms the lack of formation of ThT positive structures during the lag-phase (figure 2C). This was followed by fluorescence enhancement along the elongation-phase and it reached the equilibrium at the stationaryphase. Observation of a similar trend by LS assay that indicates the formation of higherorder structures with time further complements the conclusions drawn from sedimentation and ThT data (figure 2B and C).

The dependence of rate of aggregation on reaction concentration was established by determining the aggregation kinetics at different starting concentrations. This data showed an inverse relation between the starting concentration of the reaction and the corresponding lag-phase observed for each concentration (figure 2D). Similarly, the critical concentration observed at the equilibrium-phase was increasing upon reducing the starting concentration of the aggregation reaction (figure 2D). These observations confirm the fact that the 
efficiency of nucleation in a nucleation-dependent aggregation decreases upon reducing the starting concentration[2, 24, 34, 49, 64, 65].

The phenomenon of nucleation was further confirmed by determining the actual size of the critical nucleus formed during aggregation. For this, the aggregation kinetics at different initial concentrations was monitored (figure $3 \mathrm{~A}$ ). The initial $20 \%$ drop in the monomer concentration was used to determine the concentration $(\mu \mathrm{M})$ versus $\operatorname{time}^{2}\left(\mathrm{Sec}^{2}\right)$ curves (figure 3B). Plotting the $\log \left[\right.$ slope] of each linear time ${ }^{2}$ plot against the respective $\log [$ concentration $(\mathrm{M})]$ resulted in a linear $\log -\log$ plot with a slope of 9.2 (figure $3 \mathrm{C}$ ). This suggested a critical nucleus of size seven $\left(n^{*}=7\right.$, after round off) monomers. Collectively, these observations provide strong evidence for a nucleation-dependent aggregation mechanism for GNNQQNY[6, 10, 11, 22, 23].

The amyloid-like characteristics of mature fibrils formed at the end of aggregation were established by using different biophysical and structural characterisation methods such as FTIR, ThT, Congo-red (CR), TEM and fibre-XRD analyses (figure 4). The presence of a characteristic $\beta$-sheet band at $1631 \mathrm{~cm}^{-1}$ and a corresponding minor band at $1690 \mathrm{~cm}^{-1}$ in infrared spectroscopy data indicated the presence of $\beta$-sheet-rich structures in fibers (figure 4A). The aggregates generated through seeding of the reaction also showed similar characteristics when analysed by FTIR spectroscopy (figure 4A). These fibrils exhibited yellow-green fluorescence on staining with ThT and apple-green birefringence on staining with Congo-red dyes (figure 4B, C)[71]. The ThT fluorescence emission and CR birefringence property confirmed the presence of $\beta$-sheet-rich structures and amyloidogenic nature of fibers[50, 68, 69, 72-74]. Electron microscopy images showed long fibrous-tocrystalline structures comparable to those reported earlier for GNNQQNY (figure 4D)[28, 53, 71, 75]. Fiber diffraction analysis indicated distinct diffraction signals at $4.8 \AA$ 
(meridional) and $9.3 \AA$ (equatorial), which are characteristic signals of cross- $\beta$ architecture of amyloid fibrils (figure 4E)[3, 10, 11, 53, 71, 75].

GNNQQNY sequence was shown to induce the fibril formation in yeast Sup35 protein by constituting the fibril-core of prion fibrils $[3,76]$. These fibrils being functional in nature are usually non-toxic to the yeast cells. This was confirmed by investigating the toxicity of GNNQQNY fibers by CellRox green reagent-based oxidative stress and ReadyProbes Live/Dead cell assays. Co-incubating the differentiated SHSY5Y cells with GNNQQNY aggregates at physiological conditions $\left(\mathrm{pH} 7.4\right.$ and $\left.37^{\circ} \mathrm{C}\right)$ resulted in no significant oxidative stress levels and percentage of dead cells as compared to PBS-treated cells (figure 5A, B). This outcome was also compared with that of the known inducer of oxidative stress and cell death, $\mathrm{H}_{2} \mathrm{O}_{2}$ for confirmation[77, 78]. As shown earlier, the cytotoxicity and cell death exhibited by $\mathrm{H}_{2} \mathrm{O}_{2}$ was significantly higher as compared to GNNQQNY and PBS (figure 5A, B)[30]. Altogether, these data confirmed that the fibers formed by GNNQQNY are non-toxic in nature as reported earlier[30]. All of these evidences confirmed that the aggregates generated through the proposed solubilisation method resulted in amyloid fibers that share a great similarity with the existing published literature. These striking similarities suggested the wide applicability of this method for carrying biophysical and structural studies of amyloidogenic polypeptides.

\section{Conclusion}

In this study, we report a solubilisation protocol that generates homogenous monomeric solution. This protocol allows the monomers to remain stable until the aggregation reaction is triggered, thus facilitating the aggregation and nucleation kinetics analyses based on quantitative RP-HPLC-based sedimentation assay. The experimental results revealed that the hepta-peptide; GNNQQNY follows nucleation-dependent aggregation kinetics. The data 
under physiological conditions ( $\mathrm{pH} 7.4$ and $37^{\circ} \mathrm{C}$ ) suggested that $\sim 7$ monomers constitute to form a critical nucleus that acts as a template for further monomer addition to drive the aggregation reaction forward. The mature GNNQQNY fibers formed are long, unbranched structures and displayed apple-green birefringence and green-yellow fluorescence on staining with Congo-red and thioflavin $\mathrm{T}$ dyes, respectively. Also, these fibrils did not exhibit any increase in cytotoxicity or cell death upon co-incubating with SHSY5Y neuroblastoma cells. This study provides the basis for experimental understanding for the previous predictions which are based on theoretical and molecular dynamic simulations. Thus we believe that this solubilisation protocol will have wider practical implication to understand the kinetics and thermodynamics of aggregation of amyloidogenic sequences.

\section{Author contributions}

GB conceptualised the study, executed the experiments and interpreted the results. MBM contributed in executing the cytotoxicity and cell death studies. XRD data was recorded and analysed by GB and LCS. Manuscript was prepared by GB and edited by LCS and AKT. LCS and AKT supervised the project.

\section{Acknowledgements}

GB acknowledges the European Molecular Biology Organisation (EMBO) for providing a Short-Term Fellowship award (EMBO-STF 7674) to visit Prof. Louise C. Serpell's lab at University of Sussex, United Kingdom. LCS acknowledges funding from Alzheimer's Society in support of MBM (AS-PG-16b-010). LCS and MBM are members of the Alzheimer's Research UK South Coast Network and are grateful for their support. LCS is supported by BBSRC (BB/S003312/1). AKT acknowledges Department of Biotechnology (DBT/BSBE/20120020) and Indian Council of Medical Research (ICMR/BSBE/2016489), Govt. of India for the financial support. 


\section{Conflict of interests}

Authors declare no conflicting interests.

\section{References}

[1] Makin OS, Atkins E, Sikorski P, Johansson J, Serpell LC. Molecular basis for amyloid fibril formation and stability. Proc Natl Acad Sci USA. 2005;102:315-20.

[2] Wetzel R. Physical chemistry of polyglutamine: intriguing tales of a monotonous sequence. J Mol Biol. 2012;421:466-90.

[3] Balbirnie M, Grothe R, Eisenberg DS. An amyloid-forming peptide from the yeast prion Sup35 reveals a dehydrated beta-sheet structure for amyloid. Proc Natl Acad Sci USA. 2001;98:2375-80.

[4] Reches M, Porat Y, Gazit E. Amyloid fibril formation by pentapeptide and tetrapeptide fragments of human calcitonin. J Biol Chem. 2002;277:35475-80.

[5] Morris KL, Rodger A, Hicks MR, Debulpaep M, Schymkowitz J, Rousseau F, et al. Exploring the sequence-structure relationship for amyloid peptides. Biochem J. 2013;450:275-83.

[6] Wickner RB, Edskes HK, Bateman DA, Kelly AC, Gorkovskiy A, Dayani Y, et al. Amyloids and yeast prion biology. Biochemistry. 2013;52:1514-27.

[7] Geddes AJ, Parker KD, Atkins ED, Beighton E. "Cross-beta" conformation in proteins. J Mol Biol. 1968;32:343-58.

[8] Serpell LC, Sunde M, Fraser PE, Luther PK, Morris EP, Sangren O, et al. Examination of the structure of the transthyretin amyloid fibril by image reconstruction from electron micrographs. J Mol Biol. 1995;254:113-8.

[9] Sunde M, Serpell LC, Bartlam M, Fraser PE, Pepys MB, Blake CC. Common core structure of amyloid fibrils by synchrotron X-ray diffraction. J Mol Biol. 1997;273:729-39.

[10] Nelson R, Sawaya MR, Balbirnie M, Madsen AO, Riekel C, Grothe R, et al. Structure of the cross-beta spine of amyloid-like fibrils. Nature. 2005;435:773-8.

[11] Sawaya MR, Sambashivan S, Nelson R, Ivanova MI, Sievers SA, Apostol MI, et al. Atomic structures of amyloid cross-beta spines reveal varied steric zippers. Nature. 2007;447:453-7.

[12] Diaz-Avalos R, Long C, Fontano E, Balbirnie M, Grothe R, Eisenberg D, et al. Crossbeta order and diversity in nanocrystals of an amyloid-forming peptide. J Mol Biol. 2003;330:1165-75.

[13] Osborne KL, Bachmann M, Strodel B. Thermodynamic analysis of structural transitions during GNNQQNY aggregation. Proteins. 2013;81:1141-55. 
[14] Esposito L, Pedone C, Vitagliano L. Molecular dynamics analyses of cross- $\beta$-spine steric zipper models: $\beta$-Sheet twisting and aggregation. Proc Natl Acad Sci USA. 2006;103:11533-8.

[15] Gsponer J, Haberthür U, Caflisch A. The role of side-chain interactions in the early steps of aggregation: Molecular dynamics simulations of an amyloid-forming peptide from the yeast prion Sup35. Proc Natl Acad Sci USA. 2003;100:5154-9.

[16] Lipfert J, Franklin J, Wu F, Doniach S. Protein misfolding and amyloid formation for the peptide GNNQQNY from yeast prion protein Sup35: simulation by reaction path annealing. J Mol Biol. 2005;349:648-58.

[17] Mousseau N, Derreumaux P. Exploring the early steps of amyloid peptide aggregation by computers. Accounts of chemical research. 2005;38:885-91.

[18] Strodel B, Whittleston CS, Wales DJ. Thermodynamics and Kinetics of Aggregation for the GNNQQNY Peptide. Journal of the American Chemical Society. 2007;129:16005-14.

[19] Szała B, Molski A. Aggregation kinetics of short peptides: All-atom and coarse-grained molecular dynamics study. Biophysical Chemistry. 2019;253:106219.

[20] Szała-Mendyk B, Molski A. Clustering and fibril formation during GNNQQNY aggregation: a molecular dynamics study. Biomolecules. 2020;10:1362.

[21] Reddy AS, Chopra M, de Pablo JJ. GNNQQNY--investigation of early steps during amyloid formation. Biophys J. 2010;98:1038-45.

[22] Nasica-Labouze J, Meli M, Derreumaux P, Colombo G, Mousseau N. A multiscale approach to characterize the early aggregation steps of the amyloid-forming peptide GNNQQNY from the yeast prion Sup-35. PLoS Comput Biol. 2011;7:e1002051.

[23] Nasica-Labouze J, Mousseau N. Kinetics of amyloid aggregation: a study of the GNNQQNY prion sequence. PLoS Comput Biol. 2012;8:e1002782.

[24] Wetzel R. Kinetics and thermodynamics of amyloid fibril assembly. Acc Chem Res. 2006;39:671-9.

[25] Rubenstein R, Gray PC, Cleland TJ, Piltch MS, Hlavacek WS, Roberts RM, et al. Dynamics of the nucleated polymerization model of prion replication. Biophys Chem. 2007;125:360-7.

[26] Riesner D. Prions and their biophysical background. Biophys Chem. 1997;66:259-68.

[27] Konno H, Watanabe-Nakayama T, Uchihashi T, Okuda M, Zhu L, Kodera N, et al. Dynamics of oligomer and amyloid fibril formation by yeast prion Sup35 observed by high-speed atomic force microscopy. Proceedings of the National Academy of Sciences of the United States of America. 2020;117:7831-6.

[28] Langkilde AE, Morris KL, Serpell LC, Svergun DI, Vestergaard B. The architecture of amyloid-like peptide fibrils revealed by X-ray scattering, diffraction and electron microscopy. Acta Cryst D. 2015;71:882-95. 
[29] Burra G, Thakur AK. Unaided trifluoroacetic acid pretreatment solubilizes polyglutamine peptides and retains their biophysical properties of aggregation. Anal Biochem. 2016;494:23-30.

[30] Burra G, Thakur AK. Insights into the molecular mechanism behind solubilization of amyloidogenic polyglutamine-containing peptides. Pept Sci. 2019;111:e24094.

[31] Katz JJ. Anhydrous trifluoroacetic acid as a solvent for proteins. Nature. 1954;174:509.

[32] Jao S-C, Ma K, Talafous J, Orlando R, Zagorski MG. Trifluoroacetic acid pretreatment reproducibly disaggregates the amyloid $\beta$-peptide. Amyloid. 1997;4:240-52.

[33] Fezoui Y, Hartley DM, Harper JD, Khurana R, Walsh DM, Condron MM, et al. An improved method of preparing the amyloid $\beta$-protein for fibrillogenesis and neurotoxicity experiments. Amyloid. 2000;7:166-78.

[34] O'Nuallain B, Thakur AK, Williams AD, Bhattacharyya AM, Chen S, Thiagarajan G, et al. Kinetics and thermodynamics of amyloid assembly using a high-performance liquid chromatography-based sedimentation assay. Methods Enzymol: Academic Press; 2006. p. 34-74.

[35] Higham CE, Jaikaran ET, Fraser PE, Gross M, Clark A. Preparation of synthetic human islet amyloid polypeptide (IAPP) in a stable conformation to enable study of conversion to amyloid-like fibrils. FEBS Lett. 2000;470:55-60.

[36] Jaikaran ET, Higham CE, Serpell LC, Zurdo J, Gross M, Clark A, et al. Identification of a novel human islet amyloid polypeptide beta-sheet domain and factors influencing fibrillogenesis. J Mol Biol. 2001;308:515-25.

[37] Malavolta L, Pinto MRS, Cuvero JH, Nakaie CR. Interpretation of the dissolution of insoluble peptide sequences based on the acid-base properties of the solvent. Protein Sci. 2006;15:1476-88.

[38] Chen S, Wetzel R. Solubilization and disaggregation of polyglutamine peptides. Protein Sci. 2001;10:887-91.

[39] Ryan TM, Caine J, Mertens HDT, Kirby N, Nigro J, Breheney K, et al. Ammonium hydroxide treatment of $\mathrm{A} \beta$ produces an aggregate free solution suitable for biophysical and cell culture characterization. PeerJ. 2013;1:e73-e.

[40] Jakubek RS, White SE, Asher SA. UV Resonance Raman Structural Characterization of an (In)soluble Polyglutamine Peptide. The Journal of Physical Chemistry B. 2019;123:1749-63.

[41] Burra G, Thakur AK. Anhydrous trifluoroacetic acid pretreatment converts insoluble polyglutamine peptides to soluble monomers. Data Brief. 2015;5:1066-71.

[42] Kuipers BJ, Gruppen H. Prediction of molar extinction coefficients of proteins and peptides using UV absorption of the constituent amino acids at $214 \mathrm{~nm}$ to enable quantitative reverse phase high-performance liquid chromatography-mass spectrometry analysis. J Agric Food Chem. 2007;55:5445-51. 
[43] Burra G, Maina MB, Serpell LC, Thakur AK. Nucleation-dependent Aggregation Kinetics of Yeast Sup35 Fragment GNNQQNY. J Mol Biol. 2021;433:166732.

[44] Serpell LC, Berriman J, Jakes R, Goedert M, Crowther RA. Fiber diffraction of synthetic $\alpha$-synuclein filaments shows amyloid-like cross- $\beta$ conformation. Proc Natl Acad Sci USA. 2000;97:4897-902.

[45] Ruiz-Zamora RA, Guillaumé S, Al-Hilaly YK, Al-Garawi Z, Rodríguez-Alvarez FJ, Zavala-Padilla G, et al. The CDR1 and Other Regions of Immunoglobulin Light Chains are Hot Spots for Amyloid Aggregation. Sci Rep. 2019;9:3123.

[46] Burra G, Thakur AK. Inhibition of polyglutamine aggregation by SIMILAR huntingtin N-terminal sequences: prospective molecules for preclinical evaluation in Huntington's disease. Pept Sci. 2017;108:e23021.

[47] Saha I, Singh V, Burra G, Thakur AK. Osmolytes modulate polyglutamine aggregation in a sequence dependent manner. J Pept Sci. 2018;24:e3115.

[48] Thakur AK, Wetzel R. Mutational analysis of the structural organization of polyglutamine aggregates. Proc Natl Acad Sci USA. 2002;99:17014-9.

[49] Ferrone FA. Nucleation: the connections between equilibrium and kinetic behavior. Methods Enzymol. 2006;412:285-99.

[50] Picken MM, Herrera GA. Thioflavin T stain: an easier and more sensitive method for amyloid detection. In: Picken MM, Herrera GA, Dogan A, editors. Amyloid and Related Disorders: Surgical Pathology and Clinical Correlations. Cham: Springer International Publishing; 2015. p. 225-7.

[51] Ostapchenko VG, Sawaya MR, Makarava N, Savtchenko R, Nilsson KP, Eisenberg D, et al. Two amyloid states of the prion protein display significantly different folding patterns. J Mol Biol. 2010;400:908-21.

[52] Al-Hilaly YK, Foster BE, Biasetti L, Lutter L, Pollack SJ, Rickard JE, et al. Tau (297391) forms filaments that structurally mimic the core of paired helical filaments in Alzheimer's disease brain. FEBS Lett. 2020;594:944-50.

[53] Marshall KE, Hicks MR, Williams TL, Hoffmann SV, Rodger A, Dafforn TR, et al. Characterizing the assembly of the Sup35 yeast prion fragment, GNNQQNY: structural changes accompany a fiber-to-crystal switch. Biophys J. 2010;98:330-8.

[54] Maina MB, Bailey LJ, Wagih S, Biasetti L, Pollack SJ, Quinn JP, et al. The involvement of tau in nucleolar transcription and the stress response. Acta Neuropathol Commun. 2018;6:70.

[55] Maina MB, Bailey LJ, Doherty AJ, Serpell LC. The Involvement of A $\beta 42$ and Tau in Nucleolar and Protein Synthesis Machinery Dysfunction. Front Cell Neurosci. 2018;12:220.

[56] Maina MB, Burra G, Al-Hilaly YK, Mengham K, Fennell K, Serpell LC. Metal- and UV-catalyzed oxidation results in trapped amyloid- $\beta$ intermediates revealing that selfassembly is required for $A \beta$-induced cytotoxicity. iScience. 2020;23:101537. 
[57] Jackson M, Mantsch HH. The use and misuse of FTIR spectroscopy in the determination of protein structure. Critical reviews in biochemistry and molecular biology. 1995;30:95-120.

[58] Barth A. Infrared spectroscopy of proteins. Biochimica et biophysica acta. 2007; 1767:1073-101.

[59] Barth A. The infrared absorption of amino acid side chains. Progress in biophysics and molecular biology. 2000;74:141-73.

[60] Nelson DLNDLLALCMM. Lehninger principles of biochemistry. New York: W.H. Freeman; 2008.

[61] Branden CI, \& Tooze, J. . Introduction to Protein Structure. 2nd ed. New York: Garland Science; 1998.

[62] Shaw KL, Grimsley GR, Yakovlev GI, Makarov AA, Pace CN. The effect of net charge on the solubility, activity, and stability of ribonuclease Sa. Protein sci. 2001;10:1206-15.

[63] Bhattacharyya AM, Thakur AK, Wetzel R. Plyglutamine aggregation nucleation: thermodynamics of a highly unfavorable protein folding reaction. Proc Natl Acad Sci USA. 2005;102:15400-5.

[64] Kar K, Jayaraman M, Sahoo B, Kodali R, Wetzel R. Critical nucleus size for diseaserelated polyglutamine aggregation is repeat-length dependent. Nat Struct Mol Biol. 2011;18:328-36.

[65] Ferrone F. Analysis of protein aggregation kinetics. Methods Enzymol. 1999;309:25674.

[66] Sparrer HE, Santoso A, Szoka FC, Weissman JS. Evidence for the prion hypothesis: induction of the yeast [PSI+] factor by in vitro-converted Sup35 protein. Science. 2000;289:595-9.

[67] Haratake M, Takiguchi T, Masuda N, Yoshida S, Fuchigami T, Nakayama M. Amyloid formation characteristics of GNNQQNY from yeast prion protein Sup35 and its seeding with heterogeneous polypeptides. Colloids and Surfaces B: Biointerfaces. 2017; 149:72-9.

[68] Groenning M. Binding mode of thioflavin T and other molecular probes in the context of amyloid fibrils-current status. J Chem biol. 2010;3:1-18.

[69] Khurana R, Coleman C, Ionescu-Zanetti C, Carter SA, Krishna V, Grover RK, et al. Mechanism of thioflavin T binding to amyloid fibrils. J Struct Biol. 2005;151:229-38.

[70] Portillo A, Hashemi M, Zhang Y, Breydo L, Uversky VN, Lyubchenko YL. Role of monomer arrangement in the amyloid self-assembly. Biochimica et biophysica acta. 2015;1854:218-28.

[71] Rambaran RN, Serpell LC. Amyloid fibrils: abnormal protein assembly. Prion. 2008;2:112-7. 
[72] Yakupova EI, Bobyleva LG, Vikhlyantsev IM, Bobylev AG. Congo red and amyloids: history and relationship. Bioscience Rep. 2019;39:BSR20181415.

[73] Glenner GG. The bases of the staining of amyloid fibers: their physico-chemical nature and the mechanism of their dye-substrate interaction. Prog Histochem Cytochem. $1981 ; 13: 1-37$.

[74] Erskine E, MacPhee CE, Stanley-Wall NR. Functional amyloid and other protein fibers in the biofilm matrix. J Mol Biol. 2018;430:3642-56.

[75] Van der Wel PCA, Lewandowski JR, Griffin RG. Solid-state NMR study of amyloid nanocrystals and fibrils formed by the peptide GNNQQNY from yeast prion protein Sup35p. J Am Chem Soc. 2007;129:5117-30.

[76] Kushnirov VV, Vishnevskaya AB, Alexandrov IM, Ter-Avanesyan MD. Prion and nonprion amyloids. Prion. 2007;1:179-84.

[77] Yang W, Dunlap JR, Andrews RB, Wetzel R. Aggregated polyglutamine peptides delivered to nuclei are toxic to mammalian cells. Hum Mol Genet. 2002;11:2905-17.

[78] Thakur AK, Yang W, Wetzel R. Inhibition of polyglutamine aggregate cytotoxicity by a structure-based elongation inhibitor. FASEB J. 2004;18:923-5. 


\section{Figures}

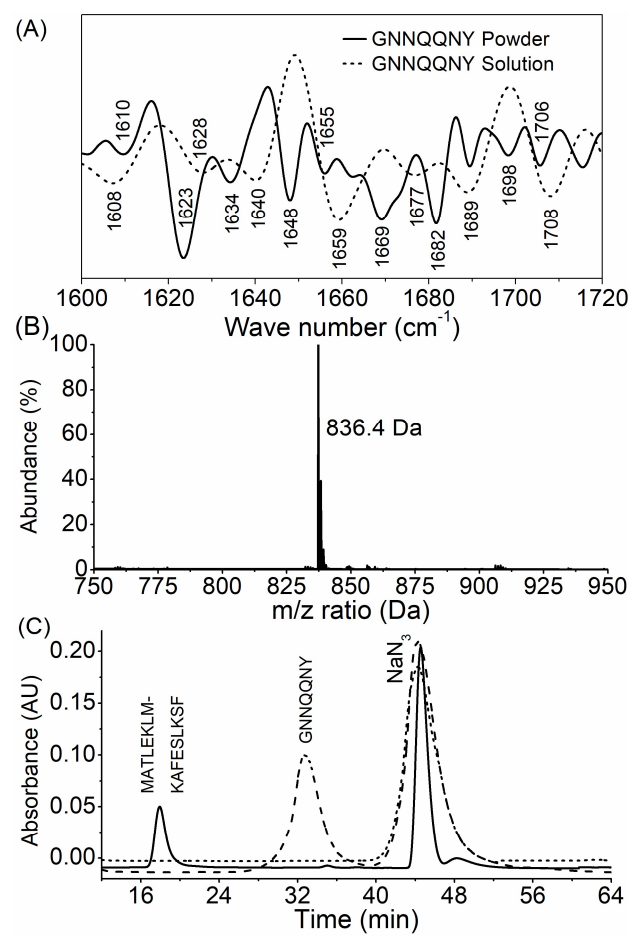

Figure 1: Biophysical characterization of GNNQQNY sequences. (A) Infrared spectroscopy analysis of GNNQQNY powder (solid line) indicating the amorphous conformation and GNNQQNY solution indicating dominant random coil conformation. (B) ESI-MS analysis showing the soluble monomeric (836.4 Da) state of GNNQQNY. (C) Size-exclusion chromatography analysis of GNNQQNY monomer (dash line), MATLEKLMKAFESLKSF (solid line) and sodium azide $\left(\mathrm{NaN}_{3}\right.$, dotted line) confirming the monomeric state of the soluble fraction. 

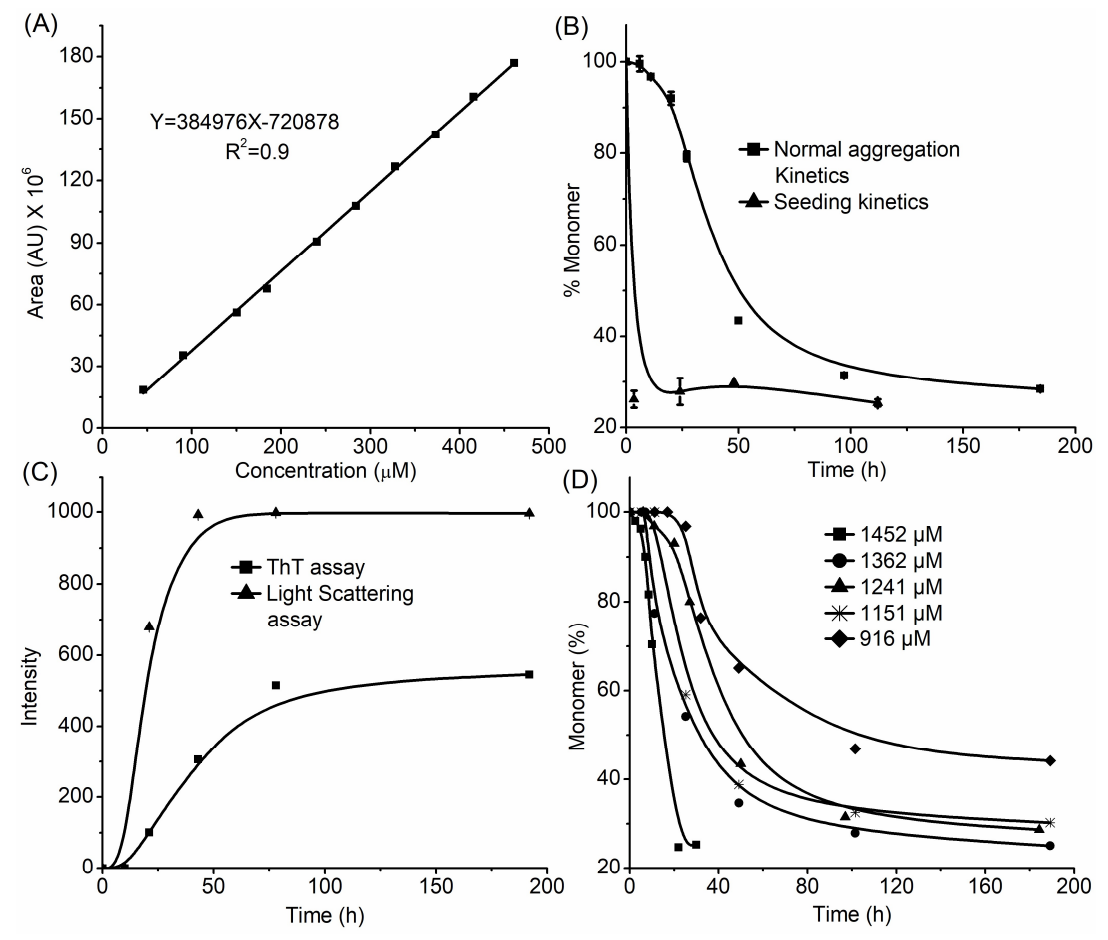

Figure 2: Aggregation analysis of GNNQQNY $(1253 \pm 12 \mu \mathrm{M})$ at $\mathrm{pH} 7.4$ and $37{ }^{\circ} \mathrm{C}$. (A) Standard curve for quantifying the monomer concentration based on sedimentation assay. (B) Aggregation kinetics analyses of normal unseeded ( $\mathbf{a})$ and seeded $(\boldsymbol{\Delta})$ reactions monitored by sedimentation assay. (C) Aggregation kinetics monitored by ThT ( $\mathbf{\Delta})$ and light scattering (a) assays showing nucleation-dependent aggregation. (D) Concentrationdependent aggregation kinetics analysis. Error bars specify the standard deviation calculated from three independent reactions.
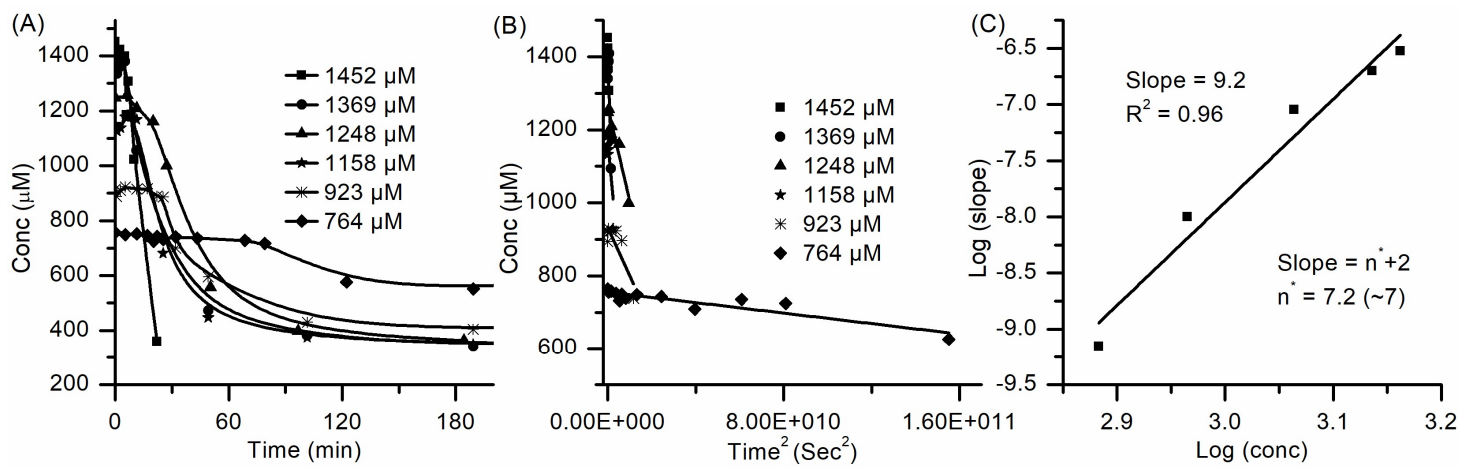

Figure 3: Nucleation kinetics of GNNQQNY at $\mathrm{pH} 7.4$ and $37{ }^{\circ} \mathrm{C}$. (A) Shows the Concentration-dependent aggregation kinetics of GNNQQNY at six different starting concentrations. (B) Plot of the initial $20 \%$ drop in the monomer against time ${ }^{2}\left(\mathrm{~s}^{2}\right)$ leading to linear time ${ }^{2}$ plot. (C) The log-log plot obtained by plotting the log[slope] obtained from (B) against the respective $\log [$ initial concentration $(\mathrm{M})]$. 

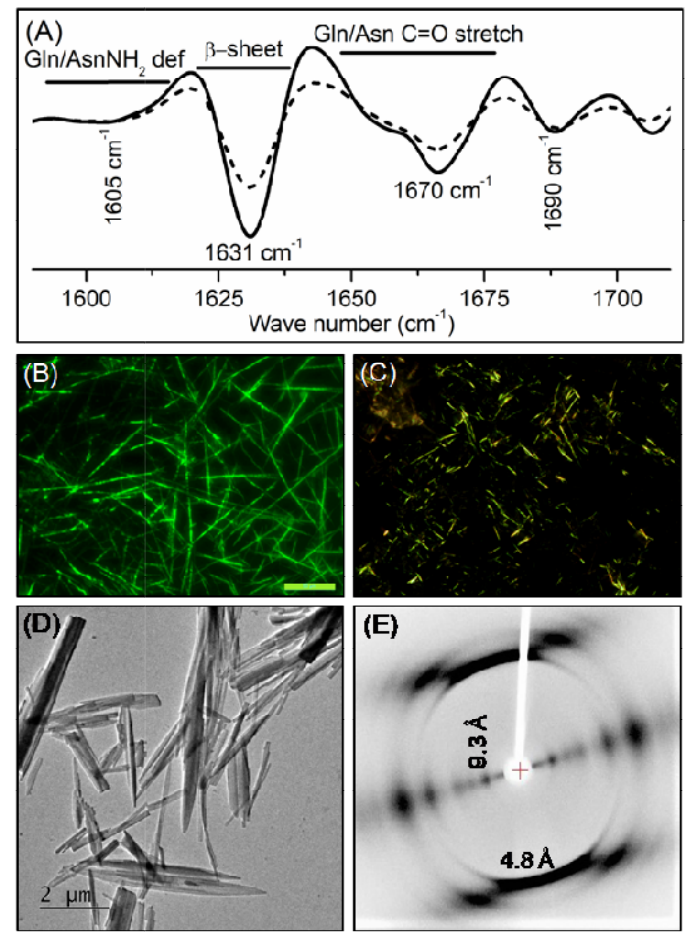

Figure 4: Biophysical and structural characterisation of GNNQQNY fibers. (A) FTIR spectra showing the characteristic $\beta$-sheet bands at $1631 \mathrm{~cm}^{-1}$ and $1691 \mathrm{~cm}^{-1}$. (B) ThT data showing distinct bright yellow-green fluorescence. (C) Congo-red staining indicating characteristic apple-green birefringence of amyloid-like structures. (D) Electron micrographs showing the fibrous-to-crystalline amyloid fibrils. (E) Fiber-XRD data showing the characteristic reflections confirming the cross- $\beta$ spine containing amyloid cores of fibers.
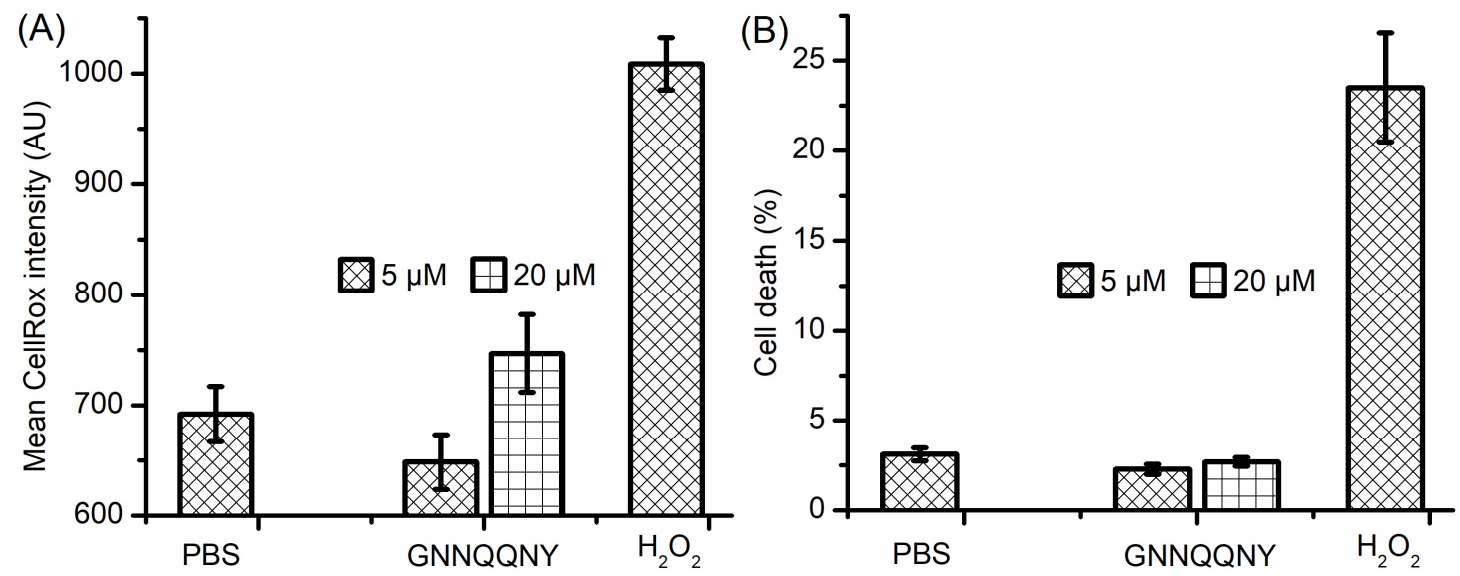

Figure 5: Toxicity of GNNQQNY fibers and controls. (A) Oxidative stress exhibited by GNNQQNY fibers, PBS and $\mathrm{H}_{2} \mathrm{O}_{2}$ on co-incubating with SHSY5Y cells for $48 \mathrm{~h}$. (B) Percent cell death observed under the same conditions. Error bars indicate the standard deviation calculated based on three independent experiments. The statistical significance $(* \mathrm{P}<0.05)$ was determined by one-way ANOVA test. 